\title{
Quaternion polar representation with a complex modulus and complex argument inspired by the Cayley-Dickson form
}

\author{
Stephen J. Sangwine* Nicolas Le Bihan ${ }^{\dagger}$
}

September 2, 2013

\begin{abstract}
We present a new polar representation of quaternions inspired by the Cayley-Dickson representation. In this new polar representation, a quaternion is represented by a pair of complex numbers as in the Cayley-Dickson form, but here these two complex numbers are a complex 'modulus' and a complex 'argument'. As in the Cayley-Dickson form, the two complex numbers are in the same complex plane (using the same complex root of -1 ), but the complex phase is multiplied by a different complex root of -1 in the exponential function. We show how to calculate the amplitude and phase from an arbitrary quaternion in Cartesian form.
\end{abstract}

\section{Introduction}

It is well-known that the complex exponential $e^{i \theta}=\cos \theta+\boldsymbol{i} \sin \theta$ generalises to quaternions by replacing $\boldsymbol{i}$ by any unit pure quaternion $\boldsymbol{\mu}$, since any unit pure quaternion is a root of $-1[6, \mathrm{pp} .203,209][5, \S 167$, p179]. Hence, any quaternion may be represented in the polar form $q=|q| e^{\mu \theta}$ where $\theta$ is a real angle [8, $\S 2.3][1, \S 12.7]$. A small difference between the complex and quaternion cases is that in the quaternion case the argument $\theta$ is conventionally confined to the interval $[0, \pi]$. This is because the modulus of the vector part of the quaternion is always taken to be positive (there is no convenient way to define an orientation in 3-space which would permit the sign of the vector part to be determined).

The classic polar form just discussed is not the only possibility for representing a quaternion. There are representations based on Euler angles using three exponentials in which the arguments of the exponentials are directly linked to the Euler angles. Bülow, for example, in [2, Theorem 2.3] and [3, §V.A] quotes the following formula, and gives an algorithm for finding the three angles:

$$
q=|q| e^{i \phi} e^{\boldsymbol{k} \psi} e^{j \theta}
$$

See also $[1, \S 12.11]$ for a discussion of Euler angles and quaternions.

The polar form we present in this paper is different to both of the preceding forms, in that it represents a quaternion using a single exponential with a complex argument and a complex 'modulus'.

\section{Polar form}

We now show that every quaternion admits the following polar form, in addition to those presented in the previous section:

$$
q=A e^{B j}
$$

where $A=a+b \boldsymbol{i}$ and $B=c+d \boldsymbol{i}$ are complex and $a, b, c$ and $d$ are real. Before proceeding, we note the analogy between this polar form, based on two complex numbers, and the Cayley-Dickson form of a quaternion $q=(w+x \boldsymbol{i})+(y+z \boldsymbol{i}) \boldsymbol{j}=w+x \boldsymbol{i}+y \boldsymbol{j}+z \boldsymbol{k}$ which is also based on two complex numbers. In the

\footnotetext{
${ }^{*}$ Department of Computing and Electronic Systems, University of Essex, Wivenhoe Park, Colchester, CO4 3SQ, United Kingdom. Email: S.Sangwine@IEEE.org

${ }^{\dagger}$ Département Images et Signal, Gipsa-lab UMR 5216, 961 Rue de la Houille Blanche, Domaine Universitaire, BP 46, 38402 Saint Martin d'Hères, Cedex, France. Email: nicolas.le-bihan@gipsa-lab.inpg.fr
} 
Cayley-Dickson form the first complex number has real and imaginary parts which are simply the first two parts of the quaternion in Cartesian form, and the second complex number has real and imaginary parts which are simply the third and fourth parts of the quaternion in Cartesian form. There is a subtlety to the Cayley-Dickson form which we must mention before proceeding, otherwise what follows may not be clear. The subtlety lies in the difference between a complex number such as $a+b \boldsymbol{i}$ and a quaternion $a+b \boldsymbol{i}+0 \boldsymbol{j}+0 \boldsymbol{k}$. In this paper, as in the classic Cayley-Dickson form, the complex number is treated as a degenerate quaternion and we handle complex numbers algebraically as quaternions in which two components happen to be zero. The usual rules of quaternion algebra therefore apply, including the rules $\boldsymbol{i}^{2}=\boldsymbol{j}^{2}=\boldsymbol{k}^{2}=\boldsymbol{i} \boldsymbol{j} \boldsymbol{k}=-1$.

The polar form presented in this paper is very subtle. It shares with the Cayley-Dickson form the idea of a construction based on two complex numbers in the same complex plane with $i$ as the root of -1 , the second of these (the argument of the exponential) being multiplied on the right by $\boldsymbol{j}$, just as in the Cayley-Dickson form. Unlike the Cayley-Dickson form, the parameters of the polar form presented here are not trivially obtainable from either the Cartesian form of a quaternion or the classic polar form.

In what follows, we first need to prove that $e^{B j}$ is a special type of quaternion, with only three components: $\alpha+\gamma \boldsymbol{j}+\delta \boldsymbol{k}$, that is, it has a zero coefficient of $\boldsymbol{i}$.

Lemma 1. Given an arbitrary quaternion in the form $p=(c+d \boldsymbol{i}) \boldsymbol{j}=c \boldsymbol{j}+d \boldsymbol{k}$, its exponential is given by:

$$
e^{p}=\cos |p|+\frac{p}{|p|} \sin |p|=\alpha+\gamma \boldsymbol{j}+\delta \boldsymbol{k}
$$

Proof. Represent $p$ by the product of its modulus and a unit quaternion obtained by dividing by the modulus:

$$
p=|p| \frac{p}{|p|}
$$

Comparison with the classic polar form of a quaternion given in $\S 1$, namely $e^{\boldsymbol{\mu} \theta}=\cos \theta+\boldsymbol{\mu} \sin \theta$, where $\boldsymbol{\mu}$ is a unit pure quaternion, is sufficient to prove the result, since $p /|p|$ is a unit pure quaternion which may be identified with $\boldsymbol{\mu}$, and $|p|$ is real and may be identified with $\theta$. Thus $\alpha=\cos |p|, \gamma=(c /|p|) \sin |p|$ and $\delta=(d /|p|) \sin |p|$.

Now we present a polar form as previously outlined with a complex 'modulus' and a complex argument, and we show how to express an arbitrary quaternion in this form, that is, how to find the complex 'modulus' and argument.

Theorem 1. Every quaternion $q=w+x \boldsymbol{i}+y \boldsymbol{j}+z \boldsymbol{k}$, where $w, x, y, z \in \mathbb{R}$ can be expressed in the form $q=A e^{B \boldsymbol{j}}$, where $A, B \in \mathbb{C}$, specifically $A=a+b \boldsymbol{i}$ and $B=c+d \boldsymbol{i}$.

Proof. The proof is by demonstrating that we can find $A$ and $B$ without any constraints on $q$. Clearly, if $q=0, A$ must be zero, and the value of $B$ is unimportant. Otherwise, we note that if we put the complex 'modulus' $A$ in polar form as $A=|A| e^{\theta i}$, we can immediately see that $|A|=|q|$. Therefore in what follows we may assume, without loss of generality, that we are working with quaternions $q$ of unit modulus, and that therefore $A=a+b \boldsymbol{i}$ is of unit modulus. Therefore we have, using the result in Lemma 1:

$$
q=A e^{B \boldsymbol{j}}=(a+b \boldsymbol{i})(\alpha+\gamma \boldsymbol{j}+\delta \boldsymbol{k})=a \alpha+b \alpha \boldsymbol{i}+(a \gamma-b \delta) \boldsymbol{j}+(a \delta+b \gamma) \boldsymbol{k}
$$

Now it is possible to see that we can obtain $a+b \boldsymbol{i}$ quite trivially, provided $\alpha \neq 0$. Assuming $\alpha \neq 0$ for the moment, and since $a+b \boldsymbol{i}$ has unit modulus, all we need is to take a complex number formed from $a \alpha$ and $b \alpha$ (the first and second components of $q$, which are the scalar part and the coefficient of $\boldsymbol{i}$ ), and normalise this complex number by dividing by its modulus. However, there is a small problem: there is an ambiguity of sign. We do not know the sign of $\alpha$, and this means we have not determined $a+b \boldsymbol{i}$ completely since $-(a+b \boldsymbol{i})$ is equally valid. It is clear that this ambiguity is insurmountable, since negating $a+b \boldsymbol{i}$ can be compensated for by negating $\alpha+\gamma \boldsymbol{j}+\delta \boldsymbol{k}$.

In the case where $\alpha=0$, but $|q|>0$, the first and second components of $q$ will be zero. Thus, $q$ is of the form $\gamma \boldsymbol{j}+\delta \boldsymbol{k}$ and we may take $a=1$ and $b=0$ (thus $A=1$ ). The factorization here is not unique, and in $\S 3$ we present an example to show this (we could have chosen $a=0$ and $b=1$, and thus $A=\boldsymbol{i}$ ). 
Once we have obtained $a+b \boldsymbol{i}$ it is simple to find $\alpha+\gamma \boldsymbol{j}+\delta \boldsymbol{k}$ by dividing $q$ on the left by $a+b \boldsymbol{i}$, or equivalently, by multiplying on the left by the inverse ${ }^{1}$ of $a+b \boldsymbol{i}$.

To obtain $B \boldsymbol{j}$ we can use the quaternion log function:

$$
B \boldsymbol{j}=\log (\alpha+\gamma \boldsymbol{j}+\delta \boldsymbol{k})
$$

Numerically, this is a simple process, since the quaternion logarithm can be computed using a complex logarithm operating on a complex number isomorphic to the quaternion. The resulting complex value can then be used trivially to construct a quaternion with vector part oriented in the same direction as the original quaternion ${ }^{2}$.

It should be clear from Lemma 1 that $B \boldsymbol{j}$ must be of the form $c \boldsymbol{j}+d \boldsymbol{k}$ and therefore the real and imaginary parts of $B$ are trivially obtained from the second and third imaginary components of $B \boldsymbol{j}$ (the coefficients of $\boldsymbol{j}$ and $\boldsymbol{k}$ ).

\subsection{Sign ambiguity}

We noted above that the sign of $A$ has an ambiguity which can be compensated by an alteration to $B$ but we did not discuss what form this alteration would take. We merely pointed out that negating $A$ required $\alpha+\gamma \boldsymbol{j}+\delta \boldsymbol{k}$ to be negated.

It is easy to see that negation of $\alpha+\gamma \boldsymbol{j}+\delta \boldsymbol{k}$ may be accomplished by adding $\pi$ to the modulus of $B$. From Lemma 1 adding $\pi$ to $|B|$ means that the angles in the cosine and sine are augmented by $\pi$ which negates their results. It is not so easy to see that the converse is not the only possibility. In fact alternative solutions exist that modify $B$ to negate $\alpha+\gamma \boldsymbol{j}+\delta \boldsymbol{k}$, and the algorithm presented above for finding $B$ using a quaternion logarithm will yield these solutions. For the present paper we confine our discussion of this issue to some numerical examples.

\section{Numerical examples}

Let $q=\frac{1}{2}(1+\boldsymbol{i}+\boldsymbol{j}+\boldsymbol{k})$. Then $|q|=1$ and $a \alpha=b \alpha=\frac{1}{2}$ which gives $A=\frac{1}{\sqrt{2}}+\frac{1}{\sqrt{2}} \boldsymbol{i}$. Multiplying $q$ on the left by the conjugate of $A$ gives $\frac{1}{\sqrt{2}}+\frac{1}{\sqrt{2}} \boldsymbol{j}$ and the quaternion logarithm gives $B \boldsymbol{j}=\pi \boldsymbol{j} / 4$ (therefore $B$ is real in this instance). To verify the result:

$$
\left(\frac{1}{\sqrt{2}}+\frac{1}{\sqrt{2}} \boldsymbol{i}\right) \exp \frac{\pi}{4} \boldsymbol{j}=\left(\frac{1}{\sqrt{2}}+\frac{1}{\sqrt{2}} \boldsymbol{i}\right)\left(\cos \frac{\pi}{4}+\boldsymbol{j} \sin \frac{\pi}{4}\right)=\left(\frac{1}{\sqrt{2}}+\frac{1}{\sqrt{2}} \boldsymbol{i}\right)\left(\frac{1}{\sqrt{2}}+\boldsymbol{j} \frac{1}{\sqrt{2}}\right)=\frac{1}{2}(1+\boldsymbol{i}+\boldsymbol{j}+\boldsymbol{k})
$$

For a second example, we take $q=1+2 \boldsymbol{i}+3 \boldsymbol{j}+4 \boldsymbol{k}$. In this case we state the result obtained numerically which is $A=2.4495+4.8990 \boldsymbol{i}$ and $B=1.1317-0.2058 \boldsymbol{i}$. The modulus of $B=1.1503$. Hence (apart from rounding errors caused by the limited precision of the intermediate results shown here):

$$
\begin{array}{r}
(2.4495+4.8990 \boldsymbol{i}) \exp (1.1317 \boldsymbol{j}-0.2058 \boldsymbol{k})= \\
(2.4495+4.8990 \boldsymbol{i})(\cos 1.1503+(0.9839 \boldsymbol{j}-0.1789 \boldsymbol{k}) \sin 1.1503)= \\
(2.4495+4.8990 \boldsymbol{i})(0.40825+0.89815 \boldsymbol{j}-0.1633 \boldsymbol{k})=1+2 \boldsymbol{i}+3 \boldsymbol{j}+4 \boldsymbol{k}
\end{array}
$$

Finally, we show an example where the value $\alpha$ in Theorem 1 is zero, and the factorization is not unique. Consider the factorization of the quaternion with value $\boldsymbol{k}$. The following two factorizations will yield this value. Firstly $A=\boldsymbol{i}$ and $B=\frac{\pi}{2}$ :

$$
\boldsymbol{i} \exp \left(\frac{\pi}{2} \boldsymbol{j}\right)=\boldsymbol{i}\left(\cos \frac{\pi}{2}+\boldsymbol{j} \sin \frac{\pi}{2}\right)=\boldsymbol{i}(0+\boldsymbol{j})=\boldsymbol{k}
$$

Secondly, $A=1$ and $B=\frac{\pi}{2} \boldsymbol{i}$ :

$$
1 \exp \left(\frac{\pi}{2} \boldsymbol{i j}\right)=\cos \frac{\pi}{2}+\boldsymbol{i} \boldsymbol{j} \sin \frac{\pi}{2}=0+\boldsymbol{i} \boldsymbol{j}=\boldsymbol{k}
$$

\footnotetext{
${ }^{1}$ The inverse of $a+b \boldsymbol{i}$ is $a-b \boldsymbol{i}$ if $a+b \boldsymbol{i}$ has unit modulus (as assumed) since the inverse is the conjugate divided by the modulus.

${ }^{2}$ See, for example, the file log.m in our Matlabß) quaternion toolbox [7]
} 


\section{References}

[1] Simon L. Altmann. Rotations, Quaternions, and Double Groups. Oxford University Press, Oxford, 1986.

[2] T. Bülow. Hypercomplex Spectral Signal Representations for the Processing and Analysis of Images. PhD thesis, University of Kiel, Germany, 1999.

[3] T. Bülow and G. Sommer. Hypercomplex signals - a novel extension of the analytic signal to the multidimensional case. IEEE Trans. Signal Process., 49(11):2844-2852, November 2001. doi: $10.1109 / 78.960432$.

[4] W. R. Hamilton. Researches respecting quaternions. Transactions of the Royal Irish Academy, 21: 199-296, 1848.

[5] W. R. Hamilton. Lectures on Quaternions. Hodges and Smith, Dublin, 1853. Available online at Cornell University Library: http://historical.library.cornell.edu/math/.

[6] W. R. Hamilton. Researches respecting quaternions. First series (1843). In H. Halberstam and R. E. Ingram, editors, The Mathematical Papers of Sir William Rowan Hamilton, volume III Algebra, chapter 7, pages 159-226. Cambridge University Press, Cambridge, 1967. First published as [4].

[7] S. J. Sangwine and N. Le Bihan. Quaternion Toolbox for Matlab®, 2005. URL http://qtfm. sourceforge.net/. Software library, licensed under the GNU General Public License.

[8] J. P. Ward. Quaternions and Cayley Numbers: Algebra and Applications, volume 403 of Mathematics and Its Applications. Kluwer, Dordrecht, 1997. ISBN 0792345134. 